\title{
Fred CONSTANT \& Justin DANIEL (dir.). Cinquante ans de départementalisation Outre-mer
}

Thierry Michalon

\section{(2) OpenEdition \\ Journals}

Édition électronique

URL : http://journals.openedition.org/plc/772

DOI : $10.4000 /$ plc. 772

ISSN : 2117-5209

Éditeur

L'Harmattan

\section{Édition imprimée}

Date de publication : 1 janvier 1998

Pagination : 342-344

ISSN : 1279-8657

\section{Référence électronique}

Thierry Michalon, «Fred CONSTANT \& Justin DANIEL (dir.). Cinquante ans de départementalisation

Outre-mer », Pouvoirs dans la Caraibe [En ligne], 10 | 1998, mis en ligne le 02 mai 2011, consulté le 22

septembre 2020. URL : http://journals.openedition.org/plc/772 ; DOI : https://doi.org/10.4000/plc.772

Ce document a été généré automatiquement le 22 septembre 2020.

(c) Pouvoirs dans la Caraïbe 


\title{
Fred CONSTANT \& Justin DANIEL (dir.). Cinquante ans de départementalisation Outre-mer
}

\author{
Thierry Michalon
}

1 Confier au nouveau-venu au CRPLC le soin de rendre compte dans cette revue du dernier en date des ouvrages collectifs de l'équipe est un excellent moyen de faciliter son intégration à celle-ci, tout en sollicitant de son regard neuf des remarques qu'une longue collaboration atténuera peut-être !

2 L'ouvrage que voici, tout d'abord, n'est pas conforme à son titre... mais on doit s'en louer. Plutôt qu'un bilan de cinquante ans de départementalisation "outre-mer ", il ne s'agit ici, à l'exception d'une contribution touchant à la Guyane, que de la Martinique. Mais " qui trop embrasse mal étreint" affirme le dicton, et ce que ce travail perd quant à l'ampleur de son objet, il le gagne par la qualité des contributions présentées, la variété des points de vue, et le tangible engagement de chacun sur un objet d'étude dont le sort, à l'évidence, le concerne personnellement. Et nous sommes loin, ici, de ces disciplines tournées sur elles-mêmes, frileusement repliées sur leurs ésotérismes, et se nourrissant des controverses doctrinales fleurissant en leur sein plus qu'elles ne sont soucieuses de faire progresser la connaissance et la construction du monde...

Un livre sur la Martinique, donc, mais un ouvrage rigoureux et complet, qui procède en trois approches successives. Un premier groupe de contributions cherche à analyser les politiques publiques conduites dans les nouveaux départements, depuis 1946, par les administrations de l'Etat, et débouche sur deux remarques centrales : en premier lieu, ces politiques ont constamment oscillé - comme le permet l'article 73 de la Constitution, qui prévoit pour les départements d'outre-mer d'éventuelles " mesures d'adaptation" - entre l'application du droit commun et des mesures dérogatoires, notamment en matière de droit social et de domaine public, engendrant des régimes juridiques souvent confus et suscitant maintes frustrations; en second lieu, les effets des politiques menées ont fréquemment démenti les objectifs poursuivis par les 
pouvoirs publics et, dès lors, rendre compte de ceux-là s'avère plus fécond, d'un point de vue scientifique, que la simple analyse de ceux-ci.

Un second groupe de contributions s'efforce de retracer, sur le demi-siècle écoulé, l'évolution de la compétition politique à la Martinique. Celle-ci a emprunté trois axes principaux. La départementalisation a, tout d'abord, érigé l'extension aux départements d'outre-mer de la législation sociale en vigueur en métropole, motivation première de la loi du 19 mars 1946 (" c'était le meilleur moyen d'améliorer rapidement le sort du peuple ", confia rétrospectivement Aimé Césaire), en enjeu central de la vie politique locale. Ensuite, ou plutôt parallèlement, le champ politique martiniquais s'est restructuré autour de l'interrogation sur l'évolution statutaire du pays, en trois principaux courants: départementaliste, autonomiste, indépendantiste. Enfin, la progression contemporaine, dans les urnes, de ce troisième courant, s'avère le fait d'un électorat jeune, citadin, et protestataire.

5 La troisième partie de l'ouvrage, Identités, culture et idéologie, s'attelle quant à elle avec un certain courage à la critique de courants d'idée pourtant prédominants parmi l'élite intellectuelle martiniquaise. On s'y attardera donc plus ici. Plusieurs contributeurs s'inscrivent en effet en faux contre certaines analyses ayant le vent en poupe, et que des phénomènes d'entraînement diffusent largement au sein de l'opinion, bien au-delà des cercles restreints les ayant engendrées.

6 Cette critique porte d'abord sur la thèse de l' "oppression culturalo-idéologique" délibérée qui serait à la racine de la dépendance croissante suscitée, au fil du demisiècle écoulé, par la départementalisation: rompant avec ce confort intellectuel, certaines pages de J.Daniel, notamment, démontrent, exemples à l'appui, que cette dépendance est surtout le produit d'une convergence objective d'intérêts à la fois nationaux et locaux. Dès lors, dénouer l'écheveau de nos intérêts serait une démarche d'émancipation certainement plus honnête et plus efficace que la dénonciation réitérée de leur oppression...

7 Une approche tout aussi décapante est empruntée pour le champ culturel. Y. Bernabé, V. Capgras et P. Murgier dénoncent en effet "la fiction d'un groupe uni dans un consensus culturel communautaire", "l'absence de confrontation artistique, l'ouverture réduite aux mouvements internationaux", "la fausse évidence d'une culture qui serait, immédiatement, unique et universelle", ainsi que "la confusion...entre le rôle social de l'action culturelle, d'une part, et la création artistique de l'autre ". Ils en appellent ainsi, très directement, en matière d'expression culturelle, à l'éveil d'un esprit critique aujourd'hui émoussé par un unanimisme " militant " voué à de redoutables impasses.

On retrouve ce regard libre dans le domaine fondamental de l'identité, surtout abordé par M. Giraud et E. Jos. Tous deux dénoncent les dangers que représente le courant de pensée actuellement prépondérant, celui de la "créolité", lorsqu'il croit trouver les fondements de l'identité martiniquaise dans un héritage culturel, dans une lignée généalogique, dans une "essence" ethnique transmise de génération en génération, selon une conception "verticale" de la nation rappelant singulièrement celle de Fichte dans son "Discours à la Nation allemande" (1808). Tous deux en appellent à E. Glissant pour affirmer que l'identité n'est pas une racine, une nature, mais une relation au monde, en perpétuelle transformation, en permanente construction, et ils en appellent aussi à Renan et à sa conception "horizontale", agrégative, politique, de l'identité nationale, fondée sur l'adhésion rationnelle à un ensemble de valeurs fondant un projet 
commun. Allant plus loin, M. Giraud affirme avec vigueur, au sujet des courants successifs de la "négritude" et de la "créolité", que "la conception essentialiste de l'identité qui (les) imprègne grève lourdement le débat idéologique et la vie politique, (...) contribue à la racialisation de l'affirmation identitaire (et) pourrait bien être le fourrier d'un ordre totalitaire (...) sous le couvert d'un populisme démagogique. " Un an et demi après la rédaction de ces lignes, on se prend à espérer qu'elles ne s'avèrent pas rapidement prémonitoires...

9 On le voit, les rédacteurs de cet ouvrage n'ont cure du prétendu "détachement scientifique" qui mure tant de chercheurs en sciences sociales dans un ésotérisme protecteur et stérilise largement leurs travaux eu égard à la demande sociale implicite qui s'exprime envers eux. "Voir clair", selon la consigne de Valéry, demeure la consigne d'une équipe plus soucieuse d'éclairer la voie que de gagner la reconnaissance d'un milieu académique trop souvent coupé du monde. Et elle s'y efforce dans ce douloureux contexte antillais : " avoir à lutter contre une part de ce qui vous constitue fondamentalement ", selon la bouleversante formule de M. Giraud. 